\title{
Conniving Challenges in Secondary Femtocell Network using DSA on White-Space Channel
}

\author{
Nikhil P. Wyawahare \\ Research Scholar \\ IEEE Member, Department of Electronics \\ Engineering \\ G. H. Raisoni College of Engineering, Nagpur, \\ India
}

\author{
S. L. Haridas, PhD \\ Professor \\ IEEE Member, Department of Electronics \& \\ Telecommunication Engineering \\ G. H. Raisoni College of Engineering, Nagpur, \\ India
}

\begin{abstract}
This paper is based on an exploitation of a femtocell as small scale network for home and office which utilized white-spaces available in spectrums. It is normally free spaces available when spectrum is not in access mode; since such free spectrum normally may present because of incomplete channel used. Now in case spectrum utilization under femtocell service user is promptly knows to be a primary user which required turning in to secondary user. Since primary user having accessing mode mostly of license frequencies, such user need to scan on Dynamic Spectrum Access technique for unlicensed frequency for communication setup under femtocell secondary network. In a proposed paper Scanning is done through controller section, heart of femtocell architecture so that Scalable and configurable property of it can be possible when data centric server system is operated using Linux base. Hence on TDM base messages and data can be routed under certain priority basis. Design challenge leaning become not to change in existing hardware of primary user; while using services provided by femtocell.
\end{abstract}

\section{Keywords}

DSA-Dynamic Spectrum Access, Femtocell-small network for home/office, TVWS-TV White-Space, IP- Internet Protocol, and MIMO- Multi input Multi output, DTE- Digital terminal equipment.

\section{INTRODUCTION}

In telecommunications, white spaces refer to mostly free space in which most of bands \& its channel considered for purposefully kept blank for future. By National and international bodies generally assign different frequencies for precise uses, and in most cases license based given to broadcast over these frequencies. This frequency allotment generates plan of frequency band, only for technical reasons like to avoid interference within same spectrum band. In this case, while the frequencies are unused it may be specifically assigned for a purpose, such as a guard band. Most regularly however, these white spaces exist naturally [1] between used channels published on $14^{\text {th }}$ November 2008 report according to FCC. Since handing over nearby transmissions to instantly adjacent channels will cause destructive interference to both. In addition to this white space assigned for technical reasons, there is also unused radio spectrum which has either never been used, or is becoming free as a result of technical changes. So that if some of the band space wants to utilize; a cognitive Radio is having solution over adaptively allocated spectrum coverage results in $10 \%$ of total spectrum.

Practically it has been tested using VHDL simulation proves a dynamic spectrum access in guard/ free band. It is assumed that the communication of primary nodes to the access point is working on a single frequency channel i.e. TV White Space.
Now the main objective of the research is to detect white space in time domain. For designing as well as for implementation, single frequency channel has been considered i.e. white space in frequency domain is assumed. In this scenario, four primary nodes and one secondary node is considered. For simulation and implementation purpose all the primary nodes are given equal probability to access the single frequency channel such as

$P_{\text {access }}=1 / 4$ (since four no. of primary nodes are considered)

Where $P_{\text {access }}$ is the probability of accessing single channel by primary node. Illustrate as per concept.

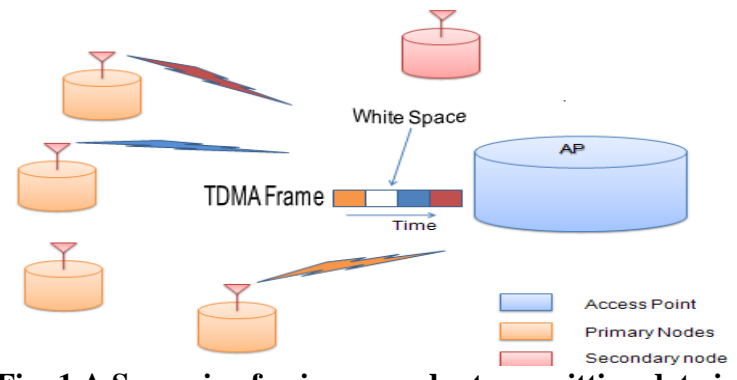

Fig: 1 A Scenario of primary nodes transmitting data in TDM mode for one frequency allocation.

To make possible equi-probable channel accessing is Time Division Multiple accessing scheme. In a main design primary and secondary nodes providing data packets, Time division multiplexing unit and a white space detection unit. [18] The primary nodes can be modeled as VHDL code for ROMs which can provide data packets. Similarly VHDL implementation of TDM and white space detection module can be designed. The white space detection module detects the white space and allots it to secondary node. In order to observe the working of implemented design the data packets are limited to eight bits and a UART module is additionally implemented to transmit the output result to the PC. Figure 2 shows how the utilization of time domain white space by a secondary node is performed.

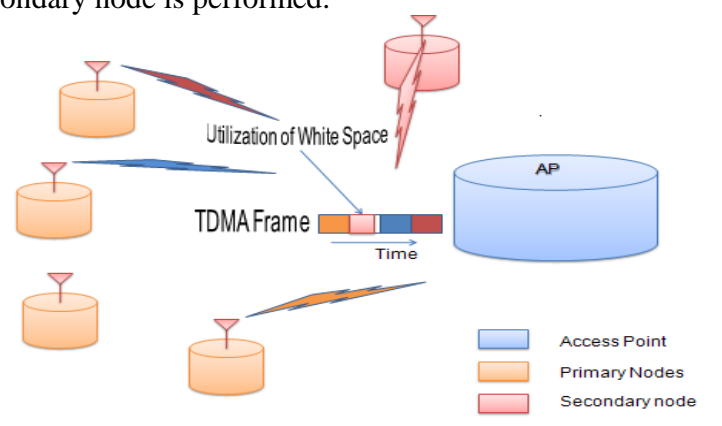

Fig: 2 Detection and utilization of White Space under one frequency. 


\section{DESIGN CONCEPT}

Primary nodes are the nodes which have primary rights to access the network service. For simulation and implementation purpose the primary nodes are modeled as message generators. The message generators are implemented by designing Simple built-in Read Only Memories ROM. A predefined 8 bit data block is stored in the ROM and a memory pointer is used to fetch consecutive data present the ROM. Four such ROMs are designed to be used to represent four primary nodes.

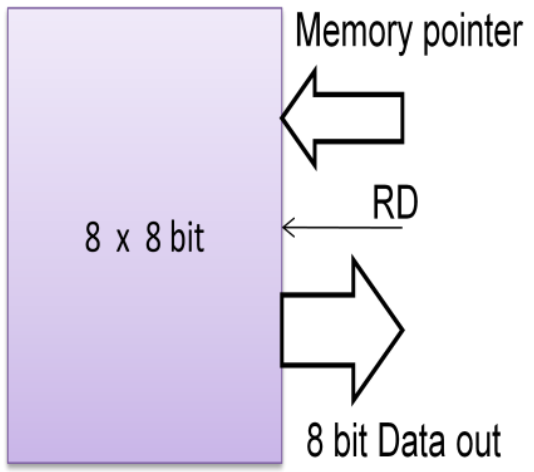

Fig: 3 In-built ROM designs for data messages controlling unit as well as memory pointer system

Message generator will transmit code as per time division base so that if blank space is to be found then it will be occupied by secondary node of terminal data. Since data is send on the UART terminal to control external hardware. UART works on the principle of asynchronous protocol. Asynchronous means that no clock signal is transmitted along the data. The receiver has a way to synchronize to the incoming data bits. Usually the 8 bit data is transmitted starting by a start bit and ended followed by the stop bit/bits. In order to transmit these data through line, RS 232 interface is used. The explanation of UART design is first started with the introduction to RS 232 protocol followed by the design of UART transmitter section module.

To make utilization of the White space by the secondary node, a UART module is designed so that the final stage output can be displayed on a personal computer PC using Windows Hyper-terminal program. Basically the Transmitter part of the UART is defined on FSM structure.

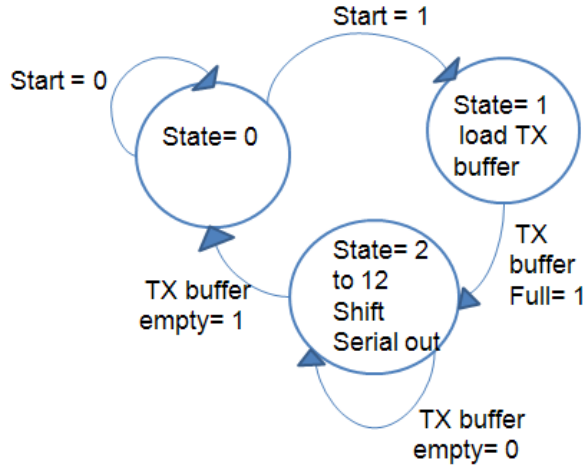

Fig: 4 State transition structures for UART transmitter section where state wise section programmed for transmission and reception.

The Finite State Machine is designed as two separate subunits namely transmitter section and State control counter. Finite state machine flow is nothing but a counter counting states i.e. state counter as shown and depending the counter's output value the task is carried out. Suppose the state counter block's initial output is STATE ' 0 ' and it encounter a rising clk edge. The state counter's output changes to STATE ' 1 '. When this value is received by the transmitter section, the transmitter buffer is loaded with the 8 bit parallel input. When the state counter's output changes to STATE '2' on rising edge of next clk pulse, then data is transferred to transmitter serial shifter unit. When the state counter counts till STATE ' 3 ', the serial shifter shifts its data present in its register by one starting from the LSB and replacing the MSB bits by a logic ' 1 '. Up to next till STATE '11' the transmitter serial shifter goes on shifting the input data and on STATE 12 a bit ' 1 ' will be present at the output.

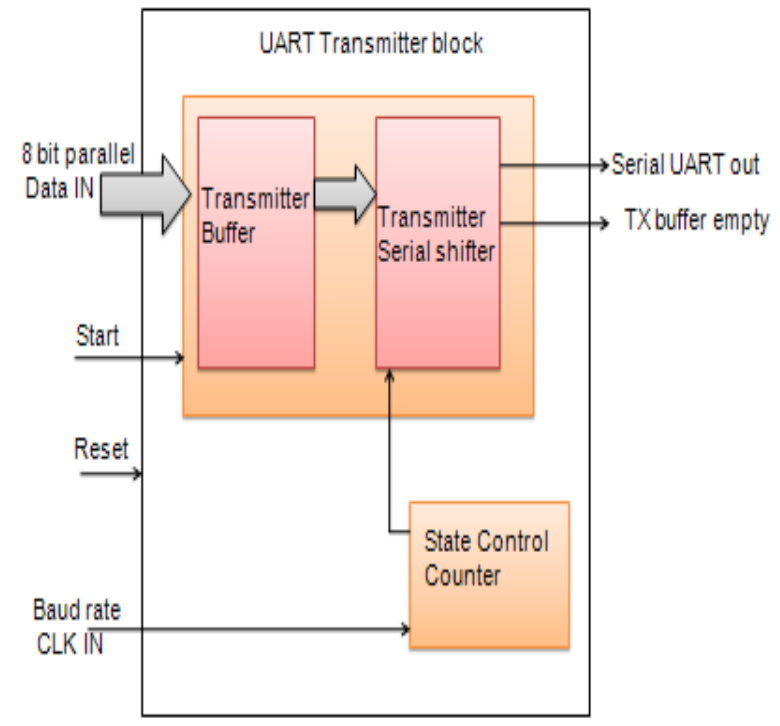

Fig: 5 Block diagram of UART transmitter section for white space utilization through 8 bit parallel data

\subsection{VHDL Implementation}

The shifter shifts the input data by replacing the previous bit locations by 1 's, so on transmitting all 8bits serially the 9th bit is ' 1 '. This logic' 1 ' represents the stop bit. The whole process is repeated again and again. To sense white-space in between two or more transmitter section and can be utilized afterword data communication. This find with testing result of RTL schematic of Message 'A' Rom unit illustrated below.

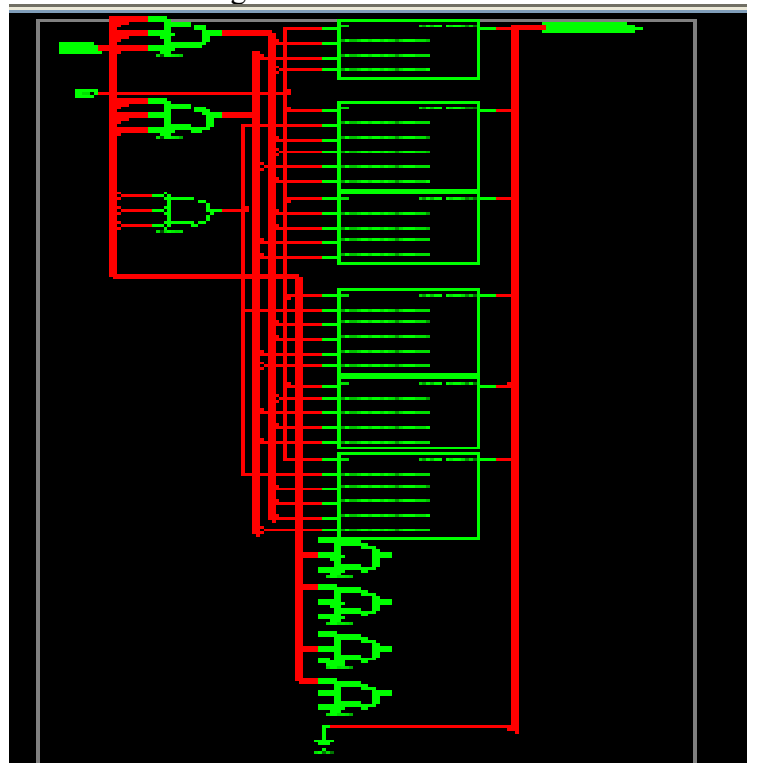

Fig: 6 RTL schematic of Message 'A' Rom unit under design unit for message transmission. 
In proposed DSA scheme the most essential design of LI_and_compare unit consists of two inputs and three outputs. This unit is designed to provide the LI values to the previous discussed block i.e. Message ' $A$ ' Rom, so that the memory's contents can be read. In addition to this, this block also provides the contents of the Message 'A' Rom to the next blocks like a latch. The value of $L I$ is incremented depending upon the positive edge trigger signal which is received from the TDM block. When the TDM block completes one frame transmission it indicates by giving a positive edge trigger at its one of the output pins. This output signal is used as a feedback to the LI_and_compare unit which increments the $L I$ pointer. The paus_out output pin is used to control the TDM operation asynchronously.

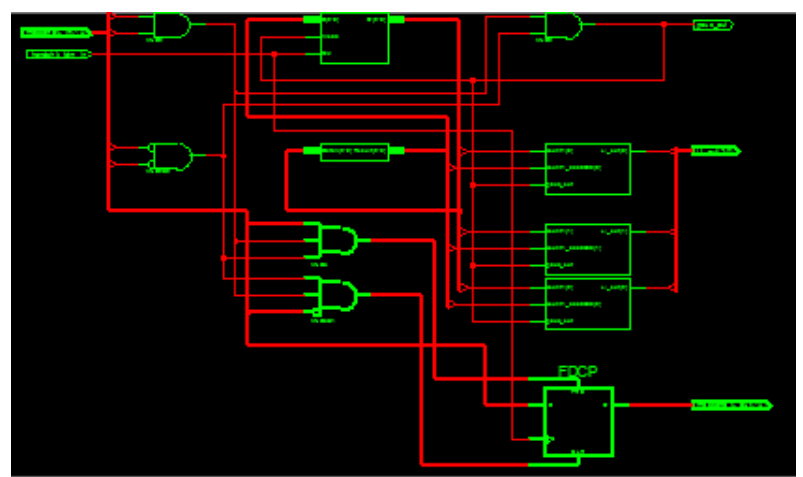

Fig: 7 RTL schematic of LI_and_compare unit, here messages compare through pointer for transmission purpose on the basis of priority

Message ' $A$ ' Rom interface with LI_and_compare unit. It makes the concurrency of system with desired change in input with TDM stable condition.

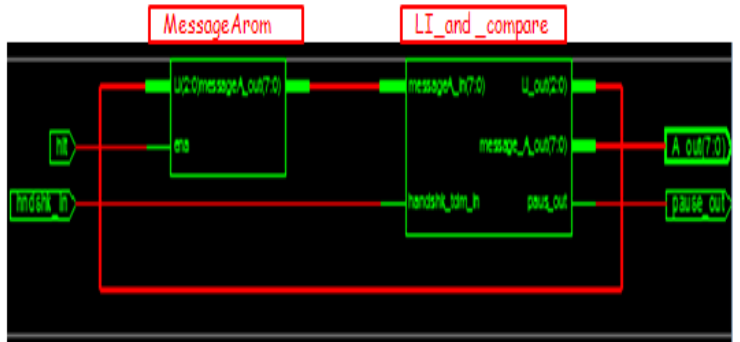

Fig: 8 RTL Structural design of Mes_gen_control unit with message unit combined with LI _comparator

\section{RESULT TESTING AND ANALYSIS}

The state counter unit is a VHDL implementation of a part of Finite state machine which counts the states like a counter and provides that at its output. Its VHDL entity name is state_cntr. As shown in Figure 9, this unit is designed to count the baud rate clock pulses and to provide counted output to the next unit named uart_txm.

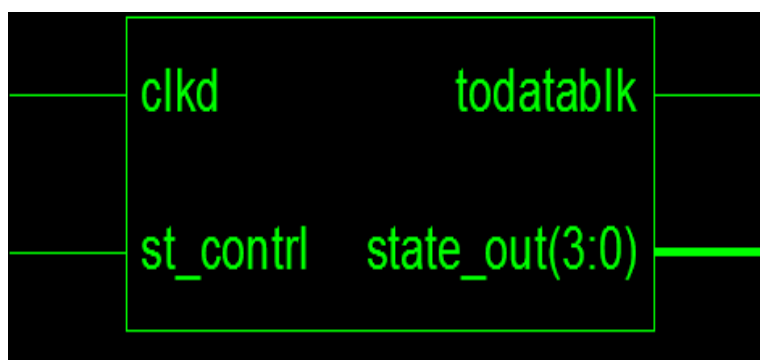

Fige: 9 synthesized design of the state_cntr unit for state selection on high priority basis.
Proposed transmitter sub-unit is named as uart_txm.vhd. The output of the state_cntr is applied to the input pin of uart_txm i.e. state. This unit is designed such as to perform operations depending upon the value received from the state_cnntr. The operation includes the data transfer from transmit buffer to the transmitter shift register. Then on each count received the transmitter shift register shifts out the data serial through its LSB position. Figure 10 shows the synthesized design of the unit uart_txm.

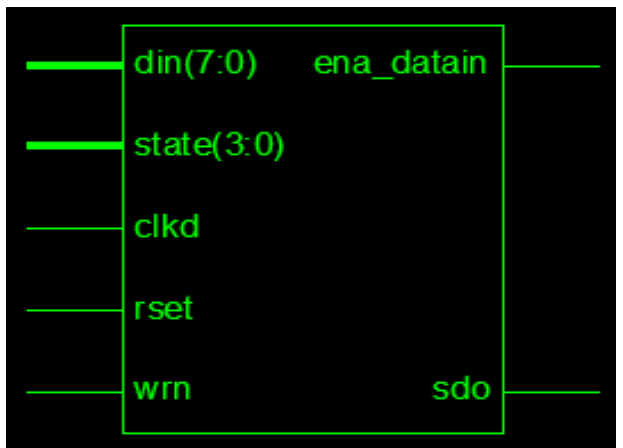

Fig: 10 Synthesized Design of uart_txm unit illustrate data input with state selection for data in enable.

Simulations of uart_txm showing data sample $55 \mathrm{H}$ being serially shifted out from pin sdo.

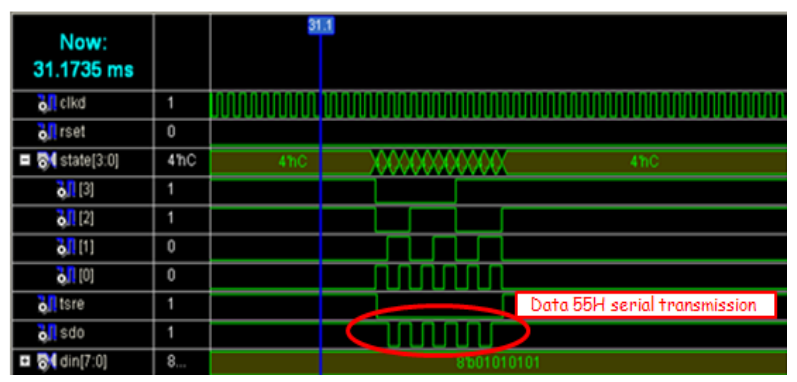

Fig: 11 Simulations of uart_txm serial shift using $55 \mathrm{H}$ serial transmission

In addition, the proposed method enables to simply adopt DSA method for detecting white space applications, allowing implementation of the proposed method at a low cost with less hardware intricacy. For to [6] Proposed that Spectrum supervision is one of the method needed for opportunistic spectrum access OSA to utilize white space without causing harmful interference to present receivers in IEEE 802.11. For an urban interference monitoring with a single node achieved more than a $4 \mathrm{~dB}$ increase of the transmit power; the Cooperative interference monitoring with 4 nodes achieved more than a $7 \mathrm{~dB}$ increase. Thus, the Interference Monitoring can expand opportunities for white space utilization without increasing the interference to the incumbent system. [7] In case of the conventional Femtocell shares the same frequency band with Macrocell, thus introducing cross-tier interference.

Results are validated with device utilization the optimization getting after reuse of slot in same time is $99 \%$. In potential different frequencies [18] band and distributed messages services required to check for optimized transmission. Following table shows the device utilization of the sub units of White space module. The delay reports generated were a total of $7.824 \mathrm{~ns}-6.103 \mathrm{~ns}$ logic, $1.721 \mathrm{~ns}$ route. 
Table: 1 Device utilization for white space

\begin{tabular}{|l|r|r|r|}
\hline \multicolumn{3}{|c|}{ Device Utilization Summary [estimated values] } \\
\hline Logic Utilization & Used & Available & Utilization \\
\hline Number of Slices & 3 & 1920 & $0 \%$ \\
\hline Number of Slice Flip Flops & 4 & 3840 & $0 \%$ \\
\hline Number of 4 input LUTs & 5 & 3840 & $0 \%$ \\
\hline Number of bonded IOBs & 3 & 173 & $1 \%$ \\
\hline Number of GCLKs & 1 & 8 & $12 \%$ \\
\hline
\end{tabular}

\subsection{Femtocell Network Challenges}

The deployment of small femtocell is big challenge to establish when coverage problems are due to multi fading signaling scheme. The released spectrum by the digital switchover may name as TV White Space (TVWS) is proposed to reduce the [16] interference for the Femtocell Network. Based on the combination of cognitive sensing and IEEE 802.11 frequency band is a novel resource use for allocation scheme is may applied to allay the interference to that Macro users are suffering from nearby Macro cell. By [8] the quantitatively investigate the feasibility of rural broadband provisioning whitespaces from the outlook of a fixed-line operator. Their techno-economical studies incorporate real white space availability; as per population density and the location of Exchanges it becomes critical design with high cost hardware to provide broad band services with basic calling services along with costs related to impractical. On the other hand use of limited IEEE 802.11 DSA spectrum bands which can be modeled into secondary user from Primary user. Secondary user can enable with subscriber identity using server user number with peer network connectivity. Every secondary user can communicate within same network on scan DSA architecture. A transmitter is works on the Omni directional antenna for typical range of 100-200 mtrs with 2.4 $\mathrm{GHz}$ single band, Here transmitter pair finds unused spectrum on the basis feature base of power optimization like many more. FCC has already open regulatory approach to used unused spectrum of $2.4 \mathrm{GHz}$.

At present works primary user spectrum is on peak research topic. Where primary user are articulate congestion to limited coverage problem. Primary user with licensed spectrum holder can use under service provider whereas secondary user is utilizing unlicensed spectrum band of complete space. All research works have prime problem statements in view of primary user regulatory services, which one of the major issue. Since potential transmitter with average probability of transmitting single operating frequency has high error. For enhancement in primary side secondary i.e. cognitive radio is having spectrum sensing [19] to utilize free spaces within secondary side is key point of research work. Proposed configuration femtocell architecture with operational range 2$2.4 \mathrm{GHz}$ ISM. In fact in $2002 \mathrm{FCC}$ initiated a practice to assess unlicensed devices to operate in the TV broadcast spectrum. [2] Basic reason is to use TV particular from 50 $\mathrm{MHz}$ to $400 \mathrm{MHz}$ is able to cover a large area and penetrate buildings, undergrowth and terrain with

Good signal. These make this technology ideal for backhaul scenario wireless communication under which fall in WhiteFi, Super Wi-Fi, Wi-Far, Wireless Regional Area Networks (WRAN). With the development of cognitive radio a highcapacity backhaul for Wi-Fi "hot spot" access points with fully linked pervasive infrastructure can easily make costeffective devices without the need for large cellular towers, providing "Internet Everywhere" capabilities.
In particular, [3] the channel allocation also depends on the specific application base, This also means that the band can be "dense" into fewer channels, while still allowing for more transmissions. The lack of prime frequencies below 2 or 3 $\mathrm{GHz}$ spectrum is a decades-old problem in wireless communication, and will be for the indefinite future. Recognizing that a static allocation of spectrum overtime and space is highly suboptimal for example, often less than $10 \%$ efficient there has been a flurry of interest in finding ways to adaptively allocate spectrum. Cognitive radio (CR) is a capable approach, where by transmitter-receiver pairs find unused spectrum (white space) and use it for communication. In Wireless applications for entertainment \& Customer service are becoming an important trend in consumer electronics domain.

Currently, [1] most of the existing applications rely on the $802.11 \mathrm{~g} / \mathrm{n}$ standards, capable to seamlessly stream HD video contents. These standards are operating in the license-free ISM band, due to which the rapid revolution in wireless communication in past years, is becoming over exploited and experiences serious coexistence problems. Here the distance problem can be solved by considering the elements that determine frequency reuse are the reuse distance and the reuse factor. The reuse distance, $D$ is calculated as,

$$
\boldsymbol{D}=\boldsymbol{R} \sqrt{ } \mathbf{3} \boldsymbol{N}
$$

Where $R$ is the cell radius and $N$ is the number of cells per cluster. By the use of MIMO system capacity can be easily increase. Now it becomes serious undertaking for a Dynamic Spectrum Access (DSA)-based cognitive users is finding spectrum ease of use. According to FCC [4] rules of action for unlicensed users in TVWS require them to register with and obtain recommendations from a list of approved Database Administrators this can design using SQL server system with data base system. Here data is only sharing between the local users which are secondary users. Server system can have authority to identify primary user who acting as secondary user under cognitive network by registering its unique identification no like mobile phone number. When user comes in Wi-Fi range of secondary network server will automatically detect user and registered it for future services like voice call and messaging. If user who becomes secondary network consumer it able to receive calls from primary network also. Since design of secondary network is to make exponential growth of wireless data with boom in multimedia applications running on smart phones, tablets, and other wireless devices. Its matter of licensed and unlicensed user application use then by the use of Wi-Fi 802.11 spectrum becomes $2.4 \mathrm{GHz}$ valuable stand to design using ISM band. Since FCC has already declared $2.4 \mathrm{GHz}$ to $5 \mathrm{GHz}$ are free to use and any one can make its own system architecture.

With cellular technology all cells becomes more overcrowding for providing stable quality of service in the field of wireless with special on demand; this will either more costly and required change in available or existing hardware of user. This may lead to slower and unplanned change in current state. White-space gives promising approach to deliver services like cellular where capacity and coverage problem is more like in rural areas. Here critical problem is to make signal availability rather than change or developing applications to user. Since all most TV bands are becoming free to use for this all application under white-space; normal user can change its hardware to access all these application is becomes chip solution over [2] deploying 3G/4G services in rural area. One major problem is to make availability of service and second is to continue all services with huge 
inflation of data tariff rates. If server manages all inter calls within small network with all supporting application like cellular in $2 \mathrm{G} / 3 \mathrm{G}$ it can become pico-cell or femtocell. So it can be a simple solution to provide free internet services and cloud data service to secondary user. Since as per research point of view Coexistence of Wi-Fi network is the common problem in the cities crowded area which can be minimized by separating frequency in between two channels about to $1 \mathrm{MHz}$ of at least. Now a day's all user are upgrading themselves into smarter phone user so to provide some educational or commercial services can becomes easy task to handle with deployment of femtocell network with $2.4 \mathrm{GHz}$ band of spectrum. Here primary user who is in dynamic nature; services which are available on primary and secondary network can be easily accessed by the time of demand

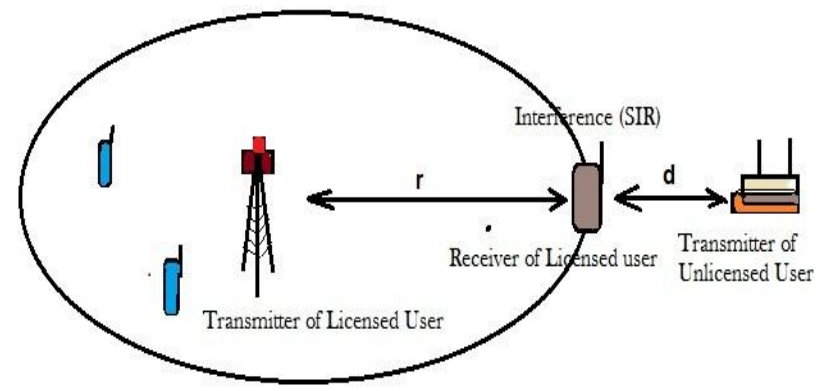

Fig: 12 Access topology design for secondary network with presence of Primary network.

This type of services can deeds like user is free to take call on both networks without any change in hardware. So to consider capacity in secondary network by the [4] Shannon capacity of a (hypothetical) secondary transmitter-receiver pair located at a point, i.e. the capacity of an infinitesimal cell with one active link where the separation between the source and receiver is negligible. The capacity is a functional part of the usual parameters - signal to noise with interference ratio and available bandwidth can be given by

$$
\text { Ccell }=\text { WO } \log 2(1+\text { SINR })
$$

Equation (2) realize capacity of one cell with average use of transreceiver pair module in (Hypothetical) condition, by the theory of channel capacity need to know Permissible Channels, Power limit, Antenna height, Interference protection for secondary area network before establishing it.

For channel utilization factor given with solution is become resulting answer to no user in one cell of secondary network that is achieved by permitting unlicensed operation and it is equal to.

\section{Available channel= 1-Unused Channels/Total Channels (3) \\ Using equation (3) easily estimated primary to secondary interface by assuming Primary transmitters are usually of very high power with poor transmission coverage. Hence [5] research topic for cognitive radio with white space utilization in pure $2.4 \mathrm{GHz}$ range according to $\mathrm{FCC}$ rule; first step is to scan all channel available in possible range by designing dynamic Spectrum Analyzer. With positive acknowledgement it's become easy moved to controller section to make decision for transmission of messages / files through Time division multiplexing towards target source.}

The spectrum overcrowding in the free bands puts in a new light the VHF and UHF bands that are currently seen as a promising alternative spectrum resource, especially in the outlook of the wide-reaching replacing from analog to digital
$\mathrm{TV}$ and in the light of the new rising dynamic spectrum access techniques. In this structure, the system relies on a mutual private approach of spectrum sensing. A practical digital global television submissive prototype has [9] been implemented on a test-bed based on commercial also called as DTT receivers in combination with software defined radio hardware devices. Extended measurements performed in a real indoor environment assessed the feasibility of the proposed system in terms of coverage and protection of the incumbent users. Figure 13 shows basic system design to build a configurable wireless platform that can be used to implement cognitive radio network architectures. Having of a commodity IEEE $802.11 \mathrm{a} / \mathrm{b} / \mathrm{g} \mathrm{Wi}$-Fi router at the physical layer and RF signal processing and IP traffic shaping circuitry. Also local wireless system needs to design for information sink. Figure 16 shows the complete proposed structure for MIMO femtocell [17] structure in this one or more transceiver connected via wirelessly to multi transceiver system. Here all stuff's are works on the wireless mode with special design applications like android, local design web-site.

Highly effective spectrum sensing algorithm for cognitive radio and white space applications [1] the proposed spectral covariance [6] sensing (SCS) algorithm exploits the different statistical correlations of the received signal and noise in the frequency domain. Test statistics are computed from the covariance matrix of a partial spectrogram and compared with a decision threshold to determine whether a primary signal or arbitrary type is present or not. Figure 14 illustrate a typical sensing unit show for desired femtocell.

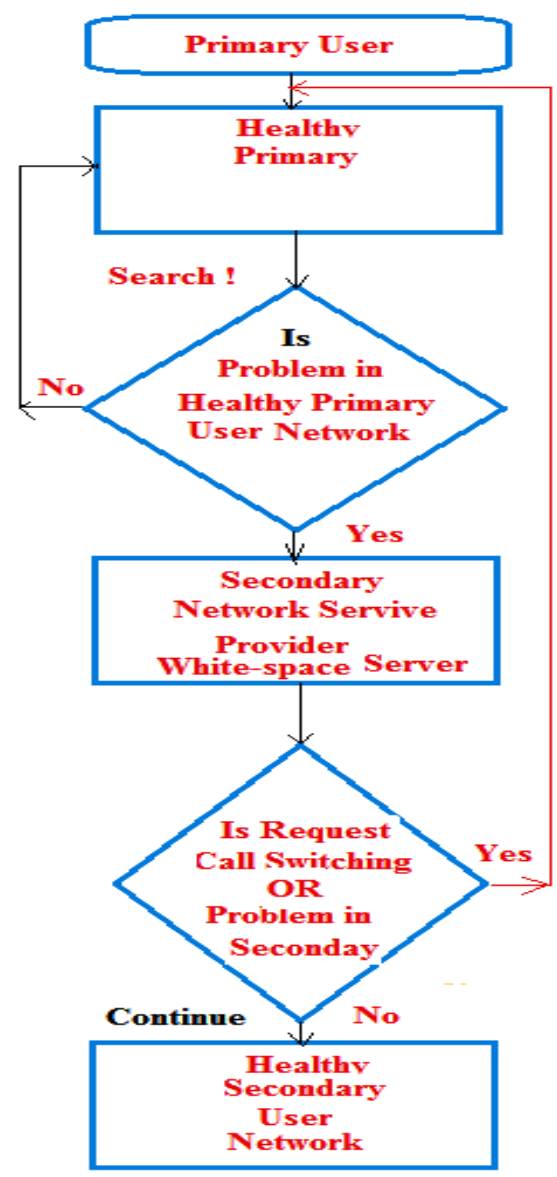

Fig: 13 Femtocell architecture flow for selecting secondary network in atypical condition of primary network. 


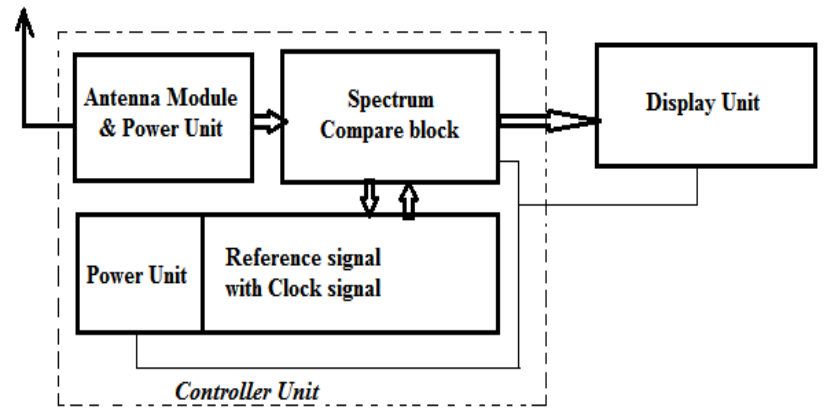

Fig: 14 Femtocell Structure for acting spectrum sensing and comparison with reference signal unit.

This detector is analyzed theoretically and verified through realistic open-source simulations using actual digital television signals captured in the US. Compared to the state of the art in the literature, SCS improves sensitivity by $3 \mathrm{~dB}$ for the same dwell time, which is a very significant improvement for this application. Further, it is shown that SCS is highly robust to noise uncertainty, whereas many other spectrum sensors are not. For this [2] it explained as White Space used in the digital TV spectrum for the purpose of broadband Wi-Fi networking. The formal standards for this are IEEE 802.11af (White-Fi) and IEEE 802.22 (Wi-Far ${ }^{\mathrm{TM}}$ ). [7] There are several very enticing advantages for reusing this bandwidth, not the least of which is the potential for digital broadband "hot spots" with a range of several kilometers. The exciting new potential of this space, yet tempered with a few technical realities. Key to success in this market will be products that have flexible, programmable and scalable architectures which provide cost-effective solutions to match the density, power and throughput.

\subsubsection{System on chip design}

Figure 15 shows new proposed System-on-Chip (SoC) devices can be used to meet these severe, and often conflicting, necessities. Are need to [3] proposed that the involvement presents four business challenges associated with a potential secondary use of European TV white spaces by LTE networks. Based on a quantitative study for 11 representative European countries, they show that Europe's TV white spaces are relatively scarce, diminishing, spatially scattered and heterogeneous, as well as rural in nature. These intrinsic characteristics of the spectrum have implications for the exploitation scenarios by secondary LTE networks that regularly appear as capable use cases. These implications relation to three LTE use case scenarios: femtocell deployments, rural coverage deployments, and LTE broadcasting/multicasting deployments. And this can be utilized only by [4] TV White Space (TV-WS) is the unused TV channels in any given location as the result of the transition to digital broadcasting designated by U.S. Federal Communications Commission (FCC) for unlicensed use. The present's significant and new opportunities within the context of emerging $4 \mathrm{G}$ networks for developing new wireless access technologies that meet the goals of the US National Broadband Plan. The model is later used to explore various trades-offs between network capacity and system parameters and suggest possible amendments to FCC's incumbent protection rules in the favor of furthering white space capacity. So to [5] the protection of incumbent users such as digital television (DTV), white space applications running on locally unused TV spectrum should be able to detect incumbent users at a low signal-to-noise ratio (SNR).

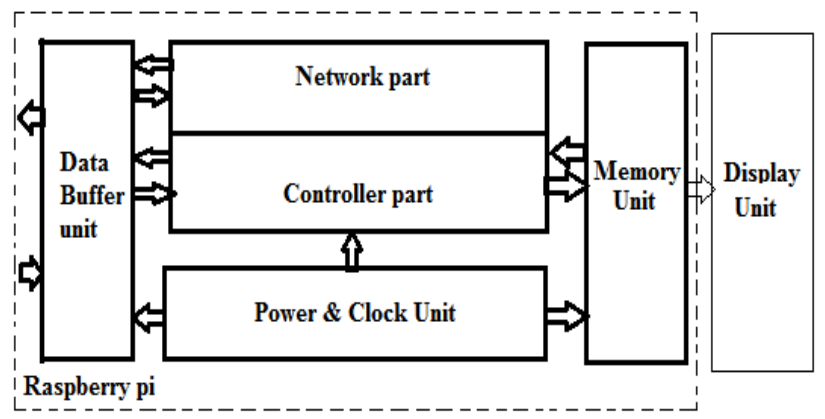

Fig: 15 System on chip design for Femtocell Structure for network routing with proper utilization of white space.

In addition, the proposed method enables them to simply adopt the DTV pilot detector for detecting white space applications, allowing implementation of the proposed method at a low cost with less hardware complexity. For to [6] Proposed that Spectrum management is one of the key functions needed for opportunistic spectrum access (OSA) to utilize white space without causing harmful interference to incumbent receivers. For an urban macro cell, the Interference Monitoring with a single node achieved more than a $4 \mathrm{~dB}$ increase of the transmit power; the Cooperative Interference Monitoring with 4 nodes achieved more than a $7 \mathrm{~dB}$ increase. Thus, the Interference Monitoring can expand opportunities for white space utilization without increasing the interference to the incumbent system. [7] In case of the traditional Femtocell shares the same licensed frequency band with Macrocell, thus introducing cross-tier interference.

\section{REQUIRED FEMTOCELL NETWORK}

The released TV spectrum by the digital switchover named TV White Space (TVWS) is proposed to reduce the interference for the LTE Femtocell Network. Based on the combination of cognitive sensing and TVWS, a new resource allotment scheme is applied to moderate the interference that Macro users are suffering from nearby Femtocells. By [8] the quantitatively investigate the feasibility of rural broadband provisioning TV whitespaces from the perspective of a fixedline operator. Their techno-economical studies incorporate real white space availability; population density and the location of Exchanges in the UK, along with costs related to infrastructure, backhaul, and sites, which were often overlooked in previous studies. The study findings indicate the provision of rural broadband in TVWS spectrum is a technically and commercially highly feasible solution. Here [9] proposed spectrum overcrowding in the free bands puts in a new light the VHF and UHF bands that are currently seen as a promising alternative spectrum resource, especially in the perspective of the worldwide switchover from analog to digital TV and in the light of the new emerging dynamic spectrum access techniques. Extended measurements performed in a real indoor environment assessed the feasibility of the proposed system in terms of coverage and protection of the incumbent system idea [10] is that to increase the system capacity of a wireless link is by getting the transmitter and receiver closer to each other, which creates the dual benefits of higher quality links and more spatial reuse. 


\section{FEMTOCELLNETWORK HARDWARE}

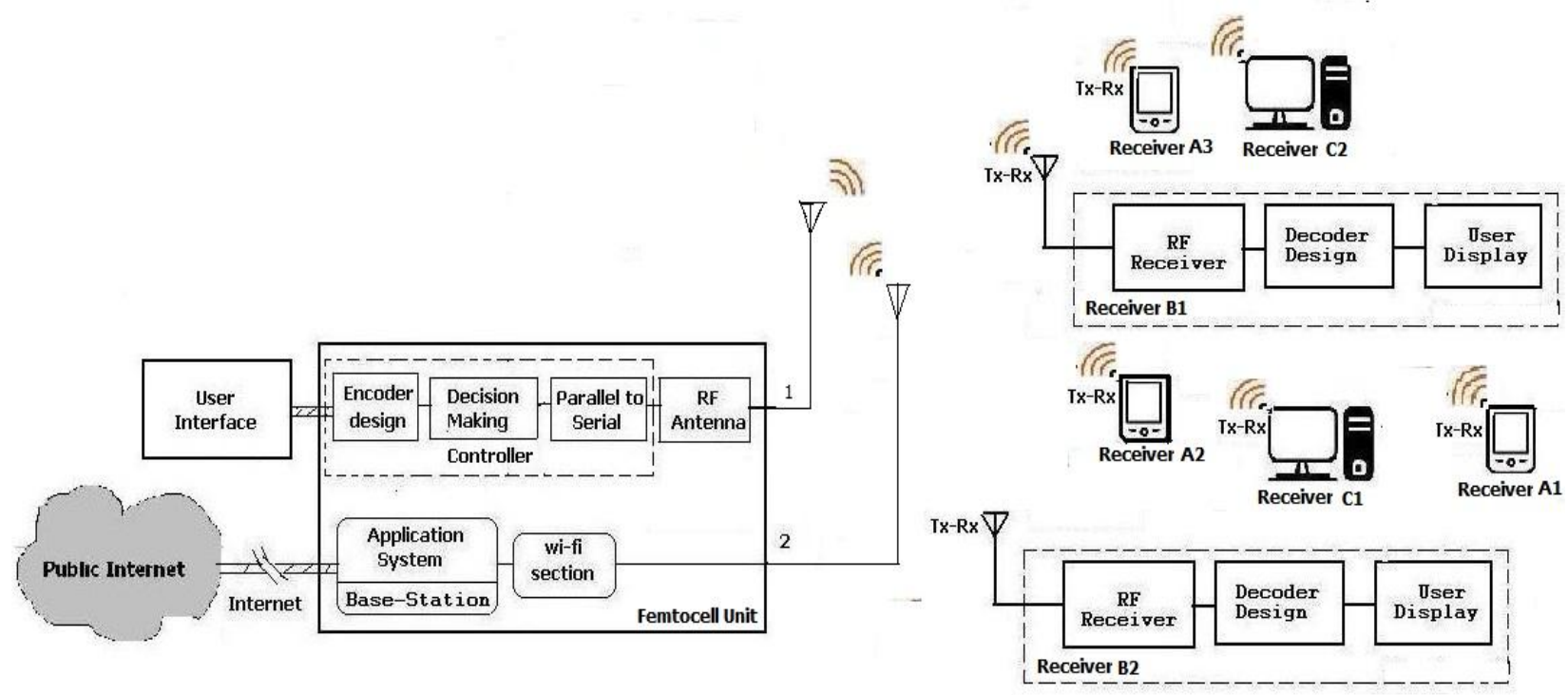

Fig: 16 Multi-input Multi-output Femtocell Structure with secondary network user field.

The recent concept of femtocells also called home basestations which are data access points installed by home users to get better indoor voice and data coverage. In this article, they overview the technical and business point of view for femtocells, and describe the state-of-the-art on each front and also describe the technical challenges facing femtocell networks, and give some preliminary ideas for how to overcome them. Using the proposed [11] concepts of intraoperator spectrum white space reuse, multi-operator spectrum sharing, and multi-service spectrum reuse in femtocells to address this problem. They describe, in detail, candidate architecture and associated novel technologies namely a cognitive femto base station, a femto coordination/controller server, a multi-operator shared spectrum server.

So currently exploring a prototype implementation of these technologies. Explained [12] as an overview of existing issues and promising cellular backhaul technologies and identified design and challenges for further research. The discussion on emerging "all-IP" network trends, femtocell networks, as well as issues of cellular backhaul over wireless mesh networks. It can be [13] proposed to utilize an idea of white spaces for a backhaul network for internet traffic based on existing restrictions. FCC requirements on sensing and avoiding harm to wireless microphones will have only a minor impact on capacity. This can be explained more by Considering a proposed [14] undertook to build a configurable wireless platform that can be used to implement cognitive radio network (CRN) architectures. So consisting of a commodity IEEE $802.11 \mathrm{a} / \mathrm{b} / \mathrm{g}(\mathrm{WiFi})$ router at the physical layer and RF signal processing and IP traffic shaping circuitry, the resultant hybrid terminal (called the WiFi CR) becomes a building block [15] that can implement cognitive femtocells, point to multipoint, mesh, and relay wireless networks. The IP addressable white wi-fi $\mathrm{CR}$ terminals can sense their radio environment, schedule IEEE 802.11 packet transmissions in space and time, and select channel, modulation rates, and transmit power.

\section{CONCLUSION}

Developed a generic cognitive radio by manipulating the operation of an off-the-shelf commodity IEEE $802.11 \mathrm{~g}$ wireless router using a combined software/hardware overlay. This is given with present proof-of concept demonstrator for an adaptive inter-system-interface gateway between LMR and IP based networks. To overcome its limitation the flexibility of the proposed solution, a satellite link is utilized to enable the integration of an independent LMR cell or direct connected terminals (MicroSpot) into an existing infrastructure over long distances without the need for dedicated transport networks. Making advantage in future to deploy such a system, a prior in depth analysis of the restrictions of the components is performed. These results are used for an overall usability analysis based on measurements with a demonstrator. Main challenge is a high variance in the delay, which inhibit a direct connection between a strictly timed TDMA LMR network and IP-based transport networks. The proposed gateway is based on an adaptive convergence layer multiplexer, which optimizes the LMR data stream for the transmission on a time variant channel like a satellite link. Spectrum utilization for secondary user can be done easily by finding free ISM band unused frequency under DSA. Since all users are not ease of use to wifi so some specific services like calling and multimedia messaging for registered user under secondary cell site femtocell can provide better opportunity where primary user can finds problems in network congestions, multipath diversity and low signaling conditions.

Due to the optimize server and intelligence network router service may avail for privet sectors like schools, offices, colleges, hospitals, rural areas where users are in same range of location around $<200 \mathrm{mts}$. These entire users can be initialize both primary and secondary network at same time only it makes difference when and whom from to do call. Since internal calling and messaging service is can be made costless to entire register secondary user if they required service it becomes its major advantage to rural area user. 


\section{ACKNOWLEDGEMENT}

I personally acknowledge the IEEE papers; spectrum published on the VANET and mobile computing IEEE spectrum issues that support me for prolific literature survey.

\section{ABOUT AUTHOR}

Author having PG in M.Tech VLSI, in 2011 from RTMNU Nagpur University, Nagpur, Maharashtra, India; Currently he is research scholar for $\mathrm{PhD}$ under ARC centre G.H.Raisoni College of Engineering, Nagpur. With 7 years of academic experience under Electronics and Telecommunication \& Electronics Engineering Department.

\section{REFERENCES}

[1] Jaeweon Kim, Student Member, IEEE, and Jeffrey G. Andrews, Senior Member, IEEE, "Sensitive White Space Detection with Spectral Covariance Sensing" in IEEE TRANSACTIONS ON WIRELESS COMMUNICATIONS 1536-1276 Volume: 9, Issue: 9, 2010.

[2] Peter Flynn,Product and program manager,Multicore processos Texas Instruments, "White SpacePotentials and Realities", January 2013.

[3] Jaap VAN DE BEEK, Janne RIIHIJ ARVI, Petri MAHONEN, "Intrinsic challenges (and opportunities) to deploy LTE in Europe's TV white spaces" in Future Network and MobileSummit 2012 Conference Proceedings, Paul Cunningham and Miriam Cunningham (Eds) IIMC International Information Management Corporation, 2012.

[4] Farzad Hessar, Student Member, IEEE, Sumit Roy, Fellow, IEEE, "Capacity Considerations for Secondary Networks in TV White Space"arXiv:1304.1785v1 [cs.NI] 28 Mar 2013.

[5] Seungil Yoon and Kyutae Lim, "Frequency-Domain Coexistence Beacon for the Coexistence of White Space Applications".978-1-4244-3574-6/10\$25.00@2010 IEEE.

[6] Kazushi Muraoka Hiroto Sugahara Masayuki Ariyoshi, "Monitoring-Based Spectrum Management for Expanding Opportunities of White Space Utilization", in 2011 IEEE International Symposium on Dynamic Spectrum Access Networks (DySPAN).

[7] Fei Peng, Yue Gao, Yue Chen, K K Chai, Laurie Cuthbert, "Using TV White Space for Interference Mitigation in LTE Femtocell Networks" in Proceedings of ICCTA2011.

[8] Santosh Kawade, Maziar Nekovee, "Is Wireless Broadband Provision to Rural Communities in TV Whitespaces Viable? A UK Case Study and Analysis" in 2012 IEEE International Symposium on Dynamic Spectrum Access Networks.

[9] Mauro Fadda, Maurizio Murroni and Vlad Popescu, Member, IEEE, “A Cognitive Radio Indoor HDTV
Multi-Vision System in the TV White Spaces", in IEEE Transactions on Consumer Electronics, Vol. 58, No. 2, May 2012.

[10] Vikram Chandrasekhar and Jeffrey G. Andrews, "Is Femtocell Networks: A Survey", in Texas Instruments June 28, 2008.

[11] Milind M. Buddhikot, Irwin Kennedy, Frank Mullany and Harish Viswanatha, "Ultra-Broadband Femtocells via OpportunisticReuse of Multi-Operator and MultiService Spectrum" in Bell Labs Technical Journal 13(4), 129-144 (2009).

[12] Orawan Tipmongkolsilp, Said Zaghloul and Admela Jukan, "The Evolution of Cellular Backhaul Technologies: Current Issues and Future Trends", in IEEE COMMUNICATIONS SURVEYS \& TUTORIALS, VOL. 13, NO. 1, FIRST QUARTER 2011.

[13] Cyrus Gerami, Narayan Mandayam, Larry Greenstein WINLAB, Rutgers University 671 Route 1 South, North Brunswick NJ 08902, "Backhauling in TV White Spaces", in IEEE Communications Society subject matter experts for publication in the IEEE Globecom 2010 proceedings.

[14] John Sydor, David Roberts, Bernard Doray and Amir Ghasemi, "A Generic Cognitive Radio Based on Commodity Hardware", in IEEE INFOCOM 2011 Workshop On Cognitive \& Cooperative Networks.

[15] Sebastian Subik, Brian Niehoefer and Christian Wietfeld, "Adaptive Multiplexing Gateway for LMR-based Group Communications over High Latency IP-based Satellite Backhaul Links".Homeland Security (HST), 2012 IEEE Conference on Technologies 13-15 Nov. 2012.

[16] Ritika R. Gundawar, Nikhil P. Wyawahare "Design of correlative non-overlapping ISI symbol transmission system over UHF/VHF Vacant Spread Spectrums" International Conference on Convergence of Technology, 978-1-4799-3759-2/14/\$31.00@2014 IEEE

[17] Abhilash P. Tembhurne, Nikhil P. Wyawahare "Alamouti Based on Spatial Modulation for Data Transmission" International Conference on Convergence of Technology, 978-1-4799-3759-2/14/\$31.00@2014 IEEE

[18] Nikhil P. Wyawahare, Dr. S.L.Haridas "Designing Challenges to utilize white-Space for Configurable femtocell base on slotted TDM" International Conference on Contemporary Computing and Informatics IC3I- 27 - 29 November 2014, 978-1-47996629-5/14/\$31.00@2014 IEEE

[19] Rijwan Y. Musani, Nikhil P. Wyawahare, Dr.S.L.Haridas "Design and Implementation of Feature Detection Based Unused Spectrum Sensing: ISM Band" IEEE 2nd International Conference on Knowledge Collaboration in Engineering, March 27- 28, 2015 978-1-4799-86194/15/\$31.00 @2015 IEEE. 